\title{
Evaluation of recombination losses in thin film solar cells using an LED sun simulator - the effect of RbF post-deposition on CIGS solar cells
}

\author{
Marc Daniel Heinemann*, Tim Kodalle, Charles Hages, Michael Klupsch, Dieter Greiner, Lars Korte, \\ Sergej Levcenco, Thomas Unold, Rutger Schlatmann, and Christian A. Kaufmann \\ Helmholtz Zentrum Berlin, 12489 Berlin, Germany
}

Received: 25 January 2018 / Received in final form: 28 April 2018 / Accepted: 21 June 2018

\begin{abstract}
Distinguishing among different electrical loss mechanisms - such as interface and bulk recombination - is a common problem in thin film solar cells. In this work, we report a $J-V$ measurement technique using different illuminating spectra to distinguish between these two recombination losses. The basic idea is to change the relative contribution of bulk recombination to the total losses of photo-generated charge carriers by generating them in different depths within the absorber layer using different spectral regions of the illuminating light. The use of modern LED sun-simulators allows an almost free design of illumination spectra at intensities close to 1 sun. The comparison of two simple $J$ - $V$ measurements, one recorded with illumination near the absorber's band-gap energy and one with light of higher energy, in combination with supporting measurements of the absorber properties, as well as device modeling, enables the extraction of the diffusion length and the interface recombination velocity. Using this technique, we show that in CIGS solar cells, an RbF post-deposition treatment does not only reduce interface recombination losses, as often reported, but also reduces bulk recombination in the CIGS absorber. Furthermore, we find that both cells, with and without RbF treatment, are dominantly affected by interface recombination losses.
\end{abstract}

Keywords: CIGS / RbF / interface recombination / bulk recombination / LED

\section{Introduction}

Within the process of optimization of solar cells it is of major importance to identify the dominant performance limiting mechanisms. This could be material inhomogeneity or non-ideal optical or electrical properties. While many of these properties can be determined via various techniques, the detailed analysis of the electrical properties can become difficult. Especially interface recombination losses at the $p / n$-junction are almost impossible to quantify and only indirect measurement techniques exist, such as temperature dependent $J-V$ measurements [1,2], which can give rough estimates of such losses. Thus, it is necessary to distinguish the other existing electrical losses such as shunting, series resistance, back contact issues and bulk recombination losses to quantify interface recombination losses. However, the determination of bulk recombination losses is not straightforward either. One possibility is the electron-microscopybased electron beam induced current technique [3], which has the disadvantage that it has to be performed on a cross-

\footnotetext{
* e-mail: maggi.heine@gmail.com
}

section of the absorber layer. This usually leads to strong deviations from the real charge carrier collection properties due to recombination at and charging of the surface layer [4]. Another approach is to measure time-resolved photoluminescence (tr-PL) collected from the absorber material [5]. The passivation of CIGS by the CdS layer has been shown with this method [6]. Especially the use of different laser wavelengths is promising to distinguish bulk and interface recombination. Howerver, it is often observed, that only semi-finished CIGS cells can be used which do not employ a full $p / n$-junction [7]. Further, charge carrier trapping and interface recombination can influence the tr-PL results [8]. The most reliable approach to get information about bulk recombination is to measure the change of the spectral external quantum efficiency (EQE) with respect to the photon energy due to their different penetration depths into the absorber layer. This works very well if charge carrier collection is poor [9]. However, to investigate state-of-the-art thin film solar cells, it is necessary to measure the EQE at high forward bias to reduce the electric field assisted charge carrier collection; this distorts the EQE measurement due to the dark DC-current. The method proposed here, measuring 


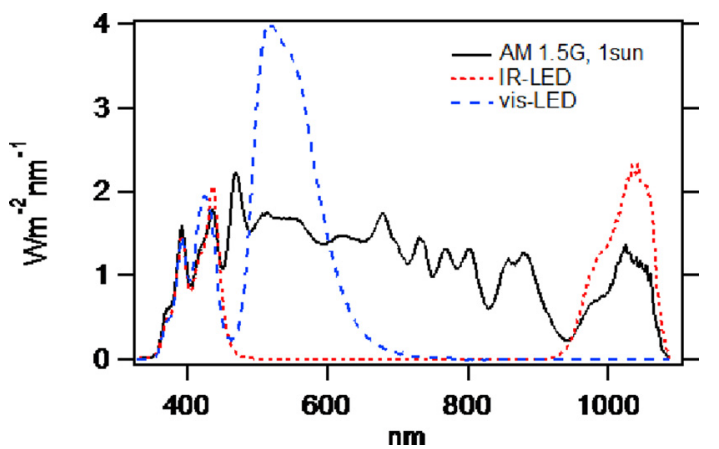

Fig. 1. Illumination spectra for the two LED setups in comparison to the AM1.5G spectrum. Note that a UV-LED is added to the IR and visible light LED.

$J-V$ curves under varying spectra of the illuminating light, is similar to the EQE method, but is more easily set-up and allows a wider voltage range, which makes it applicable to state-of-the-art solar cells. It should also be noted, that sole fitting of an illuminated and a dark $J-V$ curve does not lead to conclusive results for the surface and bulk recombination rates due to the existence of multiple solutions. We describe the details of this method and show how to use this method to investigate the influence of an RbF post-treatment on the recombination losses in CIGS solar cells.

\section{Experimental}

IV-measurements were performed using a class AAA solar simulator at 1 sun illumination intensity or, alternatively a commercial LED sun simulator at 0.3 sun illumination intensity (WaveLabs Sinus 70).

CIGS absorber layers were prepared onto molybdenum coated glass substrates using a multi-stage evaporation process as described in detail in [10]. The substrate temperature was kept at $300^{\circ} \mathrm{C}$ and $530^{\circ} \mathrm{C}$ during the first and following stages of the process, respectively. The deposition process was modified by an additional evaporation of $\mathrm{Ga}-\mathrm{Se}$ during the $\mathrm{Cu}-\mathrm{Se}$ phase. The resulting Gadepth-profile was measured by glow discharge optical emission spectroscopy (GD-OES) using a Spectruma GDA 650. To estimate the band gap energy profiles from the elemental depth profiles measured by GDOES, we used the empirical formula proposed by Ishizuka et al. [11]. CIGS layers using this deposition were prepared with and without an RbF post-deposition treatment (PDT) [12]. The PDT was performed at a substrate temperature of $280^{\circ} \mathrm{C}$ without breaking the vacuum between the CIGS and the RbF deposition. Subsequently, $50 \mathrm{~nm}$ thick $\mathrm{CdS}$ buffer layers were deposited by chemical bath deposition and $170 \mathrm{~nm}$ thick $i \mathrm{ZnO} / \mathrm{ZnO}: \mathrm{Al}$ window layers were deposited by RF-Sputtering. Finally, $\mathrm{Ni} / \mathrm{Al} / \mathrm{Ni}$ fingergrids were deposited onto the TCO layer.

Simulations were carried out using the SCAPS-1D tool developed at the University of Gent [13]. The generation profiles were generated by SCAPS using experimental values for the absorption coefficient.

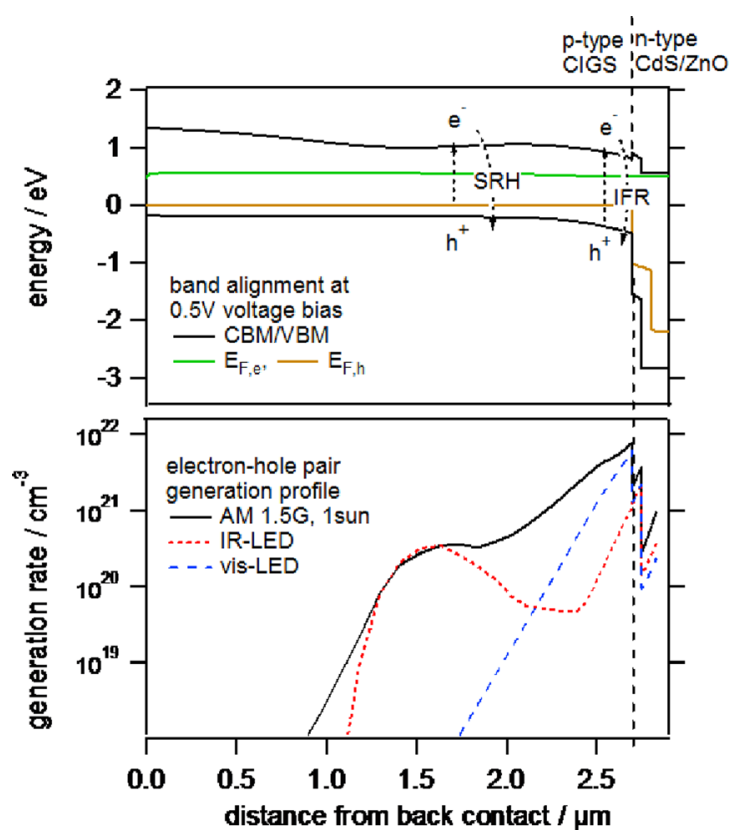

Fig. 2. Top: Energy band diagram of a CIGS solar cell similar to the one studied in this work. Possible losses are Shockley-ReadHall Recombination (SRH) in the bulk and for interface recombination (IFR). Bottom: Calculated electron-hole pair generation rate within the studied CIGS solar cell from the two LED setups used in this study in comparison with the generation rate from the AM $1.5 \mathrm{G}$ spectrum. Note that a UV-LED is added to the IR and visible light LED.

\section{Technique}

The basic idea of the method proposed in this work is to probe bulk and interface recombination losses by photo-generating electron-hole pairs at different depths within the absorber by different illuminating spectra (Fig. 1). The principle of this technique is shown schematically in Figure 2 for a CIGS solar cell with CdS buffer layer and $\mathrm{ZnO}$ window layer. The energy band profile of the CIGS device as used for the simulations in this work is shown next to the profile of the generation rate for the different illumination spectra. While most of the visible light (vis-LED) is absorbed close to the $p / n$-junction at the CdS/CIGS interface, theinfrared light (IR-LED) is absorbed deeper within the absorber layer. The photocurrent generated by the IR-LED is therefore more influenced by bulk recombination while the photocurrent generated by the vis-LED is influenced stronger by interface recombination.

The average generation depth (at which $63 \%$ of the total electron-hole pairs have been generated) within the absorber is $140 \mathrm{~nm}$ for the visible light LED (vis-LED) and $1000 \mathrm{~nm}$ for the infrared light LED (IR-LED). It should be noted that for both setups a UV-LED was added, which creates electron-hole pairs within the CdS buffer. We found that this is necessary for CIGS solar cells in terms of the stability and reproducibility of the method. We assume that these photo-generated charge carriers fill defect states within the CdS buffer layer [14]. 


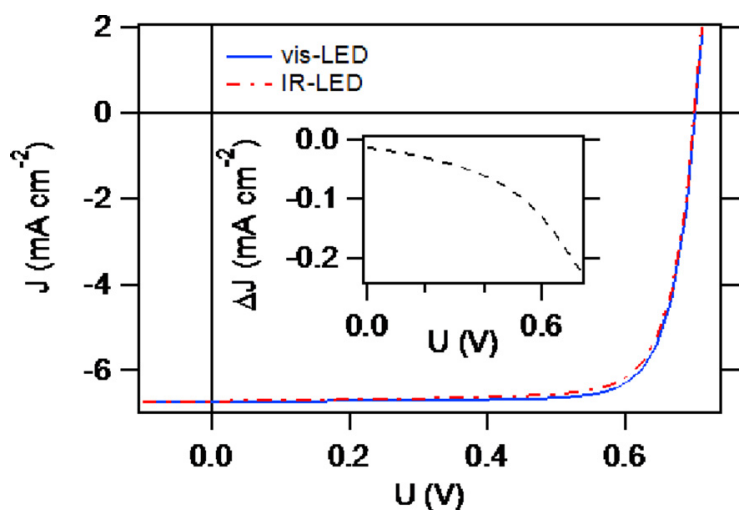

Fig. 3. Comparison of the simulated $J-V$ curves for a standard CIGS solar cell under illumination with visible and infrared light ( 0.25 suns). The inset shows the difference of the two $J-V$ curves $(\Delta J)$. The simulation uses a standard CIGS device model [13] with double graded CIGS, notch position at $500 \mathrm{~nm}$ from the $p / n$ junction, electron mobility of $100 \mathrm{~cm}^{2} / \mathrm{Vs}$ and a doping density of $N_{\mathrm{A}}=1.5 \mathrm{e}+15 \mathrm{~cm}^{3}$. In this example the diffusion length was set to $2.4 \mu \mathrm{m}$ (equivalent to $30 \mathrm{~ns}$ electron lifetime). The interface recombination velocity was set to $1 \mathrm{e}+3 \mathrm{cms}^{-1}$. As the origin of the defect states within the bulk and at the interface are not known, it was chosen to use neutral defect states.

An example of a set of simulated $J-V$ curves of a solar cell limited by bulk recombination via neutral bulk defects is shown in Figure 3. Under short-circuit conditions the photocurrents obtained from the vis-LED and the IR-LED illumination are almost equal. In spite of the different carrier generation profiles of the two illumination spectra, the large width of the space charge region at short circuit conditions leads to an efficient collection of all photo-generated minority carriers. However, with increasing applied voltage, the width of the space charge region becomes smaller and minority carriers created outside the space charge region have a higher chance of recombination. This leads to the increasing difference of the two photocurrents with increasing voltage, as shown in the inset of Figure 3. The top graph of Figure 4 shows how the photocurrent difference is influenced by the electron lifetime. A typical minority charge carrier bulklifetime for CIGS is $60 \mathrm{~ns}$, as measured with tr-PL on devices with Mo back contact and CdS buffer layer [15]. However, the magnitude of this difference depends not only on the minority carrier diffusion length but also on the Ga depth profile and the doping density of the CIGS layer. The absence of a band-gap profile leads to lower spatial separation of the IR and visible light induced average carrier generation depths, which reduces the photocurrent difference. A lower doping of the absorber leads to lower photocurrent difference as well, since the collection of charge carriers generated deeper within the absorber becomes more efficient. A very low doping density, below $10^{14} \mathrm{~cm}^{-3}$, leads to efficient collection even at voltages approaching $V_{\text {oc }}$, making the determination of the diffusion length difficult.

In case of severe interface recombination losses, above the $10^{3} \mathrm{~cm} / \mathrm{s}$ of state-of-the-art CIGS, the difference of the two photocurrents is also influenced by interface recombination. This is due to the increased hole concentration at the $p / n$-junction interface in case of electron-hole pairs
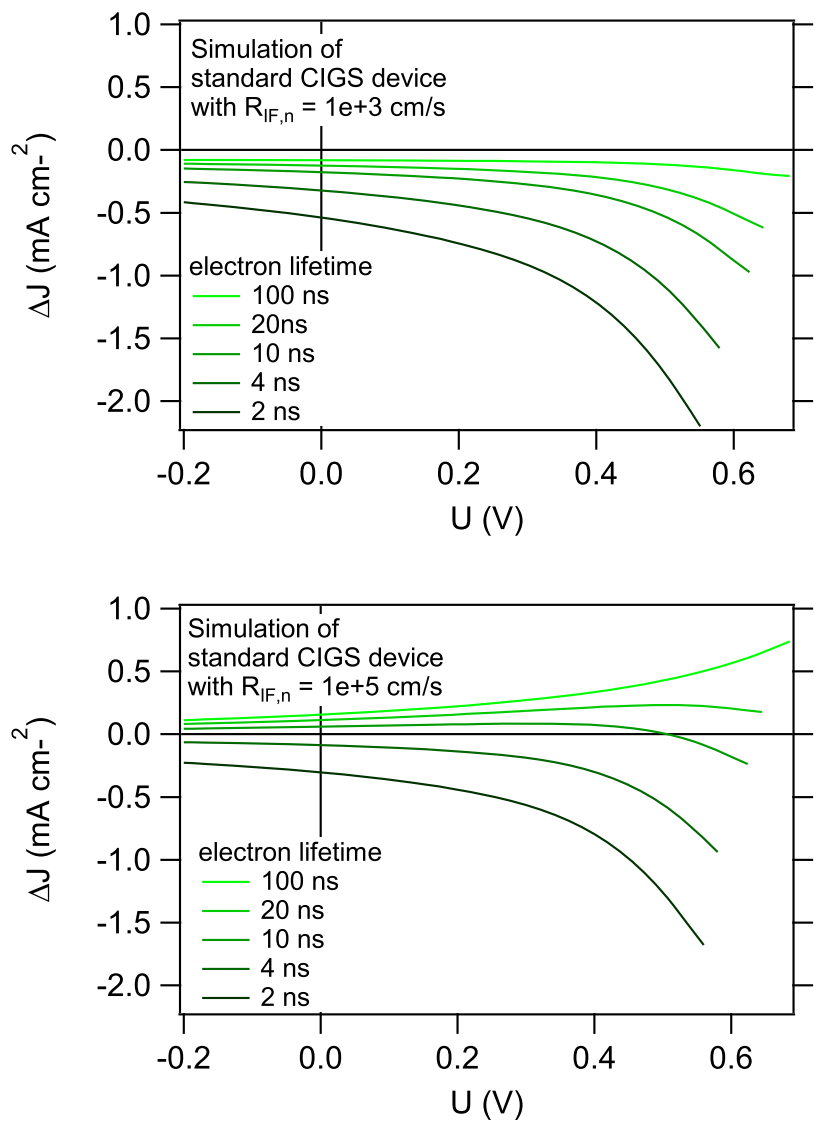

Fig. 4. Simulation of the difference between IR and visible light generated photocurrent. Device simulation parameters as described in Figure 3. Top: interface recombination velocity $R_{\mathrm{IF}, \mathrm{n}}=10^{3} \mathrm{~cm} / \mathrm{s}$. Bottom: $R_{\mathrm{IF}, \mathrm{n}}=10^{5} \mathrm{~cm} / \mathrm{s}$.

generated close to the junction-interface. The comparison of the top and bottom graphs of Figure 4 shows how the photocurrent difference is influenced by interface recombination. For low bulk recombination, it is even possible to achieve higher photocurrents with IR illumination compared to visible light illumination, resulting in an increasing positive $\Delta J$ with increasing applied voltage, up to $V_{\text {oc }}$ and beyond.

Thus, if one of the two channels is negligible, comparing $J-V$ data with both visible and IR illumination allows a very easy and quick analysis of severe bulk or interface recombination losses within a thin film solar cell. If this is not the case, it requires device modelling to separate the two effects, which in turn entails analysis of the charge carrier and band gap profile. For CIGS, the band gap profiles have to be measured for each device, since the band gap profile depends on the $\mathrm{Ga}$ and $\mathrm{Cu}$ elemental depth profiles, which can vary between different CIGS layers. To approximate the doping densities a capacitance-voltage $(C-V)$ analysis can be used $[16,17]$.

\section{Experimental results}

To enhance the spatial separation of the vis-LED and IRLED induced photo-generated charge carriers within the absorber and thus to enhance the separation of bulk and 


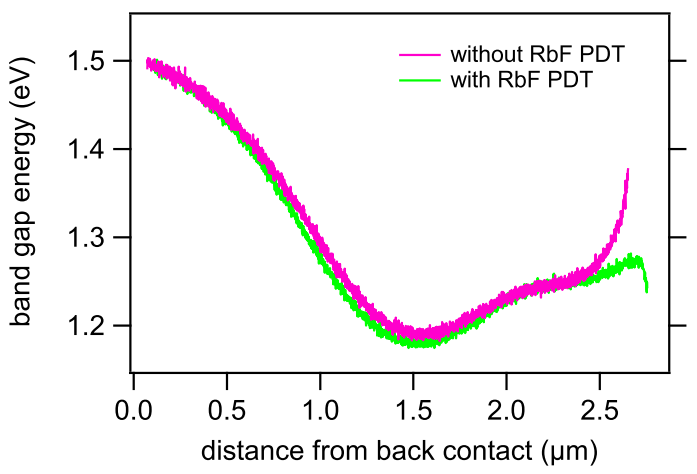

Fig. 5. Band gap profile (obtained from GDOES measurements) of the devices with and without RbF-PDT.

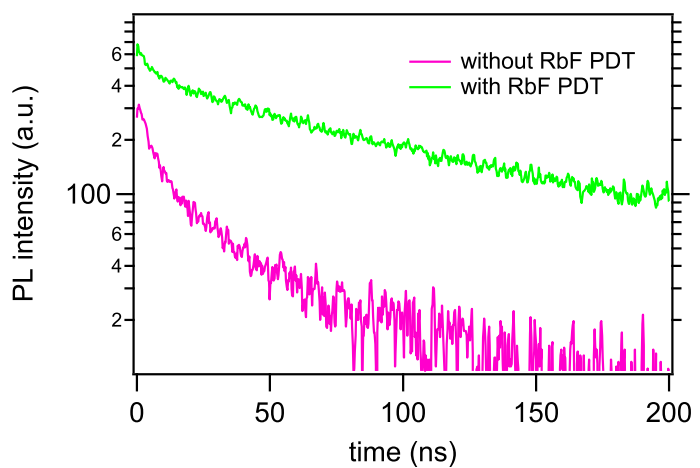

Fig. 6. tr-PL signals recorded on glass/Mo/CIGS/CdS stacks at an illumination power of $0.02 \mu \mathrm{W}$, wavelength of $660 \mathrm{~nm}$ and spot size of $30 \mu \mathrm{m}$.

interface recombination a band gap profile with a minimum band-gap (notch) position comparatively deep within the absorber was chosen as a model system for the experimental in-depth analysis. Two absorbers were prepared using identical deposition recipes, and one of them was posttreated with RbF. It was reported previously, that an $\mathrm{RbF}$ treatment leads to a reduction of recombination losses [12].

The band gap profile as shown in Figure 5 was calculated from the $\mathrm{Ga} /(\mathrm{Ga}+\mathrm{In})$ depth-profile, measured by GDOES. The notch position is $1.3 \mu \mathrm{m}$ below the film surface in both cases. It should be noted, that the $\mathrm{Ga} /(\mathrm{Ga}+\mathrm{In})$ ratios at the surface differ substantially between both samples, which we attribute to the RbF PDT, as this was the only difference between these otherwise identical deposition runs. The different surface composition is confirmed by Raman spectroscopy, as shown in Figure S1 in the Supplementary Material.

The time-resolved PL signals are shown in Figure 6. In-depth analysis of the data, as described in [18], results in an electron lifetime of $65 \mathrm{~ns}$ and a doping density of $4 \times 10^{15} \mathrm{~cm}^{-3}$ for the non-treated layer and an electron lifetime of $210 \mathrm{~ns}$ and a doping density of $5 \times 10^{15} \mathrm{~cm}^{-3}$ for the RbF-treated layer. The PL spectra recorded from both layers are shown in Figure S2. The exponential slope of the PL signal gives the sub-gap tail energy [19], which is mainly influenced by shallow defects and compositional disorder [10]. It is $25 \mathrm{meV}$ for the non-treated layer and $20 \mathrm{meV}$ for

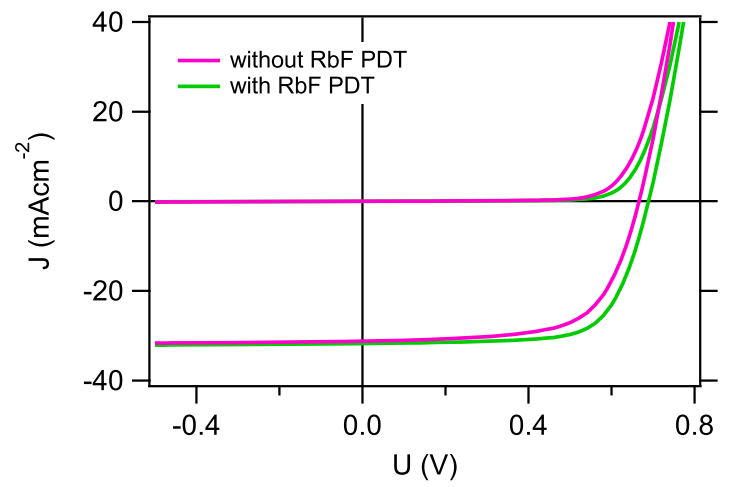

Fig. 7. $J-V$ curves of the devices with and without RbF-PDT recorded at 1 sun AM1.5G illumination.

the $\mathrm{RbF}$ treated layer. The doping density extracted from the $C-V$ measurement [17], shown in Figure $\mathrm{S} 3$, is $10^{15} \mathrm{~cm}^{-3}$ for the non-treated device and $8 \times 10^{14} \mathrm{~cm}^{-3}$ for the RbF-treated device.

The $J-V$ curves of the two devices are recorded under 1 sun illumination using the AM1.5G spectrum, shown in Figure 7. Both devices have a short-circuit current density of $31.7 \mathrm{~mA} / \mathrm{cm}^{2}$ (compare with EQE results in Fig. S4). The non-treated device has a $V_{\text {oc }}$ of $666 \mathrm{mV}$ and a fill factor of $63.7 \%$ compared to $689 \mathrm{mV}$ and $69.9 \%$ of the $\mathrm{RbF}$ treated device. The $J-V$ curves recorded under IR-LED and vis-LED spectra are shown in Figure 8. They will be discussed in the next section.

\section{Simulation and discussion}

Using a standard CIGS device model within SCAPS [20], combined with the experimental input from GDOES, $C-V$, and reflection measurements, we reproduced the $J-V$ curves obtained by illuminating the devices with the two different light spectra, as shown in Figure 8. To obtain a good fit to the experimental curves, only the defect density at the CIGS/CdS interface and in the CIGS bulk were varied. While a large number of solutions can be obtained for fitting a single $J-V$ curve, the range of possible solutions becomes much narrower when fitting two curves obtained with different illuminating spectra (this range defines the errors in Fig. 8). In this case, only the recombination mechanisms (bulk neutral defects and uniformly distributed donor and acceptor interface defects) were used as fitting parameter. The simulation results are shown together with the experimental curves in Figure 8. The non-treated device is simulated with a diffusion length of $1.6 \mu \mathrm{m}$, equivalent to a lifetime of $10 \mathrm{~ns}$ assuming an electron mobility of $100 \mathrm{~cm}^{2} / \mathrm{Vs}$, and an interface recombination velocity of $5 \times 10^{5} \mathrm{~cm} / \mathrm{s}$. The $\mathrm{RbF}$ treated device is simulated with a diffusion length of $4.1 \mu \mathrm{m}$, equivalent to a lifetime of $67 \mathrm{~ns}$, and an interface recombination velocity of $4 \times 10^{4} \mathrm{~cm} / \mathrm{s}$. Even though the values for the electron lifetime are smaller than the ones measured with tr-PL (see Tab. S1), the experimental results are in agreement with the trend of smaller electron lifetimes for the non-treated device. Further both models reproduce well the $J-V$ curves recorded at 1 sun AM1.5G 

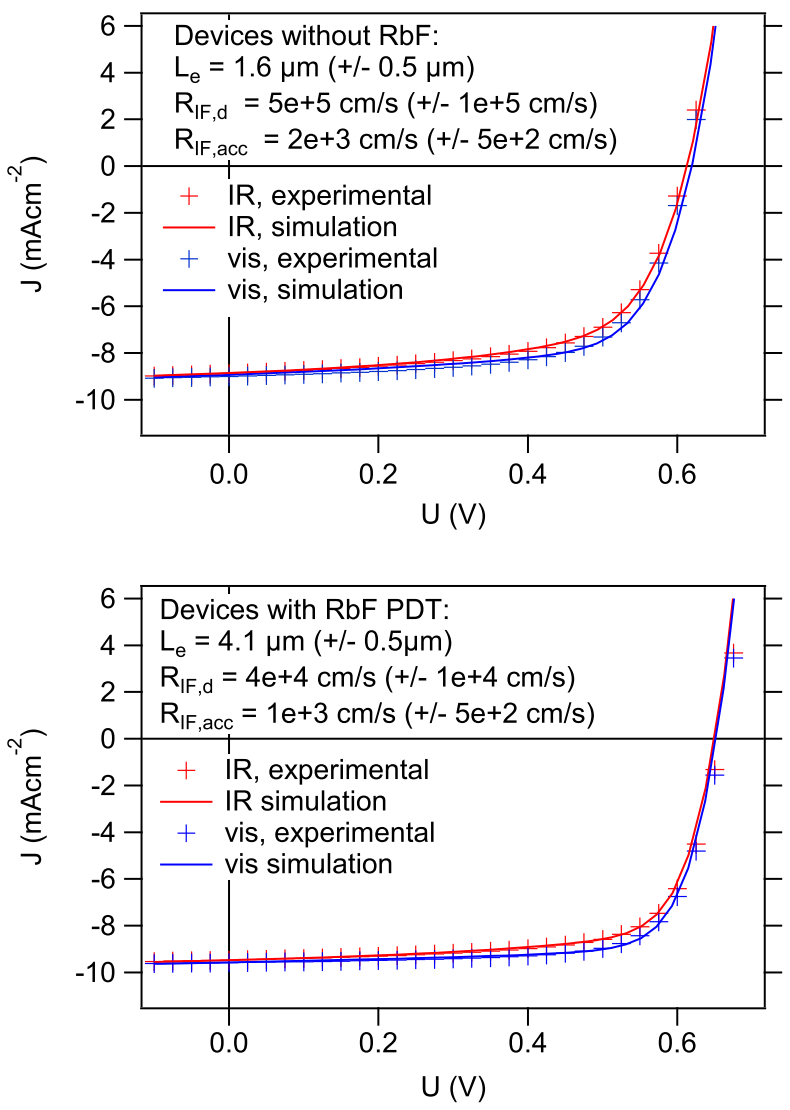

Fig. 8. Experimental and simulated $J-V$ curves with visible and infrared LED illumination. Assuming an electron mobility of $100 \mathrm{~cm}^{2} / \mathrm{Vs}, L=4.1 \mu \mathrm{m}$ relates to a lifetime of $67 \mathrm{~ns}, L=1.6 \mu \mathrm{m}$ relates to a lifetime of $10 \mathrm{~ns}$.

(compare Fig. S5 with Fig. 7). It should be noted that the experimentally derived band gap profiles of the cells were used for the simulations and the different results obtained for the treated and non-treated devices do not originate from the different band-gaps at the surface, as shown in Figure S5 (right). The experimentally observed increased electron lifetime for the RbF-treated device alone is also not sufficient to reproduce the differences. Only by changing the surface and the bulk recombination rates in the device model, it is possible to reproduce the observed difference between the $J-V$ curves of the two samples. Thus, it seems that the RbF PDT reduces the interface recombination velocity and the bulk recombination rate at the same time. While discussing the reduced interface recombination is beyond the scope of this work, it can be speculated that the reduced bulk recombination rate is connected to the observed lower sub-gap tail energy in the PL spectra, which may originate from a reduced concentration of defects in the bulk or at the grain boundaries (Fig. S2). The reduced doping density may also lead to a better charge carrier collection, however this effect is already covered by the device model.

Further, for our standard CIGS solar cells, whose notch position is positioned $500 \mathrm{~nm}$ from the $p / n$-junction, similar effects as described for the cells studied in this work can be observed (Fig. S6). However, these effects are much less pronounced due to the lower spatial separation of the photo-generated electron-hole pairs' generation depths for the two illumination conditions.

\section{Conclusion}

The proposed IV-measurement technique of using two different illuminating spectra was shown to allow a quick check for the presence of severe bulk recombination losses in CIGS and other solar cells. A detailed analysis by employing a device model showed that this technique allows to differentiate between bulk and interface recombination losses. However, it should be noted that in case of highly absorbing materials like CIGS, this analysis works only for graded absorbers or for absorbers with sufficiently low diffusion lengths. Applied to an RbF treated CIGS device it is capable of showing that the RbF treatment does reduce the interface recombination, as expected, but also decreases the bulk recombination rate.

\section{Supplementary Material}

Figs. S1-S6 and Table S1.

The Supplementary Material is available at https://www. epj-pv.org/10.1051/epjpv/2018006/olm.

\section{Author contribution statement}

M.D Heinemann: Measurements, Interpretation and Author, T. Kodalle: Fabrication of CIGS layers, C. Hages: tr-PL measurements, M. Klupsch: Raman measurements, D. Greiner: Fabrication of CIGS layers, L. Korte: Setup of LED-IV, S. Levcenco: PL measurements, T. Unold: Discussion and Interpretation, R. Schlatmann: Discussion and Interpretation, C.A. Kaufmann: Setup of CIGS lab. Discussion and Interpretation.

\section{References}

1. U. Malm et al., Thin Solid Films 480, 208 (2005)

2. J.V. Jian et al., Electrical characterization of interface recombination and its dependence on band offset, potential barrier height, and inversion in certain heterojunction solar cells, in Photovoltaic Specialist Conference (PVSC), 2014 IEEE 40th (IEEE, 2014), pp. 0686-0690

3. R. Scheer et al., Sol. Energy Mater. Sol. Cells 49, 299 (1997)

4. B. Bissig et al., Small 12, 5339 (2016)

5. A. Redinger et al., Appl. Phys. Lett. 110, 12210 (2017)

6. S. Shirakata, T. Nakada, Phys. Stat. Sol. C 6, 1059 (2009)

7. J. Chantana et al., Curr. Appl Phys. 17, 461 (2017)

8. W.K. Metzger, I.L. Repins, M.A. Contreras, Appl. Phys. Lett. 93, 022110 (2008)

9. C.J. Hages, N.J. Carter, R. Agrawal, J. Appl. Phys. 119, 014505 (2016)

10. M.D. Heinemann et al., Sci. Rep. 7, 45463 (2017)

11. S. Ishizuka et al., Jpn. J. Appl. Phys. 44, L679 (2005)

12. P. Jackson et al., Phys. Stat. Sol. RRL 10, 583 (2016)

13. M. Burgelman, P. Nollet, S. Degrave, Thin Solid Films 361, 527 (2000) 
14. T. Eisenbarth et al., J. Appl. Phys. 110, 094506 (2011)

15. A. Redinger et al., Appl. Phys. Lett. 110, 122104 (2017)

16. J.T. Heath, J.D. Cohen, W.N. Shafarman, J. Appl. Phys. 95, 1000 (2004)

17. J. Hllibrand, R.D. Gold, Determination of the impurity distribution in junction diodes from capacitance-voltage measurements, in Semiconductor Devices: Pioneering Papers (1991), pp. 191-198
18. C.J. Hages et al., Adv. Energy Mater. 7, 1700167 (2017)

19. S. Siebentritt et al., Sol. Energy Mater. Sol. Cells 119, 18 (2013)

20. K. Decock, J. Lauwaert, M. Burgelman, Energy Procedia 2, $49(2010)$

21. S. Roy et al., Mater. Chem. Phys. 73, 24 (2002)

22. J.K. Larsen et al., J. Appl. Phys. 118, 035307 (2015)

Cite this article as: Marc Daniel Heinemann, Tim Kodalle, Charles Hages, Michael Klupsch, Dieter Greiner, Lars Korte, Sergej Levcenco, Thomas Unold, Rutger Schlatmann, Christian A. Kaufmann, Evaluation of recombination losses in thin film solar cells using an LED sun simulator - the effect of RbF post-deposition on CIGS solar cells, EPJ Photovoltaics 9, 9 (2018) 